\title{
BMJ Open Longitudinal changes in
}

neurodevelopmental outcomes between

18 and 36 months in children with prenatal triptan exposure: findings from the Norwegian Mother and Child Cohort Study

\author{
Mollie E Wood, ${ }^{1,2,3}$ Jean A Frazier, ${ }^{2}$ Hedvig M E Nordeng, ${ }^{3,4}$ Kate L Lapane $^{2}$
}

To cite: Wood ME,

Frazier JA, Nordeng HME, et al. Longitudinal changes in neurodevelopmental outcomes between 18 and 36 months in children with prenatal triptan exposure: findings from the Norwegian Mother and Child Cohort Study. BMJ Open 2016;6: e011971. doi:10.1136/ bmjopen-2016-011971

- Prepublication history and additional material is available. To view please visit the journal (http://dx.doi.org/ 10.1136/bmjopen-2016011971).

Received 21 March 2016 Revised 29 June 2016 Accepted 15 July 2016

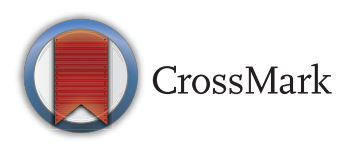

For numbered affiliations see end of article.

\section{Correspondence to} Dr Mollie E Wood; mollie.wood@gmail.com

\section{ABSTRACT}

Objective: This study sought to determine whether changes in neurodevelopmental outcomes between 18 and 36 months of age were associated with prenatal exposure to triptan medications, a class of 5-HT receptor agonists used in the treatment of migraine.

Method: Using data from the Norwegian Mother and Child Cohort Study, a prospective birth cohort that includes nearly $40 \%$ of all pregnancies in Norway from 1999 to 2008, we identified 50469 mother-child dyads who met inclusion criteria and were present for at least one follow-up assessment at 18 or 36 months postpartum. Neurodevelopment was assessed using the Child Behaviour Checklist, the Emotionality, Activity, and Shyness Questionnaire, and the Ages and Stages Questionnaire. We used generalised estimating equations to evaluate change from 18 to 36 months for children prenatally exposed to triptans, relative to contrast groups, and used marginal structural models with inverse probability of treatment and censoring weights to address time-varying exposure and confounding as well as loss to follow-up.

Results: Among eligible participants ( $n=50469), 1.0 \%$ used a triptan during pregnancy, $2.0 \%$ used triptans prior to pregnancy only, $8.0 \%$ reported migraine without triptan use and $89.0 \%$ had no history of migraine. Children with prenatal triptan exposure had greater increases in emotionality ( $\mathrm{r}-\mathrm{RR} 2.18,95 \% \mathrm{Cl} 1.03$ to 4.53) and activity problems (r-RR $1.70,95 \% \mathrm{Cl} 1.02$ to 2.8) compared to children born to mothers who discontinued triptan use prior to pregnancy.

Conclusion: Prenatal triptan exposure was associated with changes over time in externalising-type behaviours such as emotionality and activity, but not with internalising-type behaviours.

\section{INTRODUCTION}

Migraine is a chronic pain condition that affects $\sim 20 \%$ of women of reproductive age. $^{12}$ Treatment options for migraine are

\section{Strengths and limitations of this study}

Prospective, longitudinal cohort study with extensive data on exposure to medications as well as other confounders.

- Internationally recognised and extensively validated neurodevelopmental outcome measures.

- Longitudinal statistical analysis making use of multiple outcome measurement.

- No information on confounding by migraine severity or on dose or formulation of triptan medication.

- As with all observational studies, cannot rule out unmeasured confounding.

primarily pharmacologic and include analgesic medications as well as triptans, which are taken in response to an oncoming migraine episode and act to prevent or shorten the severity or duration of the migraine attack. Triptans are class of serotonin $5-\mathrm{HT}_{1 \mathrm{~B}}, 5-\mathrm{HT}_{1 \mathrm{D}}$ and $5-\mathrm{HT}_{1 \mathrm{~F}}$ receptor agonists that act on smooth muscle as well as the trigeminal cervical complex, and are the most common prescription acute migraine medication. ${ }^{3}$ To date, 10 studies have examined the safety of triptan use during pregnancy, but most of them have focused on pregnancy and very early life outcomes. Triptans have been associated with preeclampsia, preterm birth and increased risk of folate-deficient anaemia, but not with major congenital malformations. ${ }^{4-7}$ A recent meta-analysis found no increased risk of preterm birth or congenital malformation associated with prenatal triptan exposure but did note an increased risk of spontaneous abortion. ${ }^{8}$ An additional review of migraine treatment during pregnancy recommends 
that triptans may be used conservatively in pregnancy if adequate pain relief is not achieved through acetaminophen alone. ${ }^{4}$

Despite the plausibility of triptans as a neurodevelopmental teratogen, ${ }^{9}{ }^{10}$ studies of the effect of prenatal triptan on childhood neurodevelopment have been sparse. Our previous work found that prenatal exposure to triptans was associated with an increased risk of externalising behaviour in 3-year-old children ${ }^{11}$ and no associations with motor skills, communication or temperament, after adjustment for migraine severity. ${ }^{12}$ No prior studies have examined changes in behaviour over time following prenatal exposure to triptans. Since brain development is a dynamic process, examining differences in change over time may yield important insights into the mechanism by which triptans affect the developing brain.

Building on our previous research, this study aims to quantify the effect of triptan use during pregnancy on the differences in neurodevelopment outcomes from 18 to 36 months between children. Among neurodevelopmental outcomes associated with triptan use during pregnancy, we also sought to determine whether timing of triptan exposure (first trimester, second/third trimester) is related to differences in change over time.

\section{METHODS}

\section{Norwegian Mother and Child Cohort Study}

Between 1999 and 2008, the Norwegian Institute of Public Health invited women to participate in the Norwegian Mother and Child Cohort Study (MoBa). ${ }^{13}$ Women were invited prior to their first routine ultrasound appointment (gestational weeks 13-17). A total of 108841 women consented to participate (participation rate $42.7 \%$ ), with $84.8 \%$ of the participants completing the 6-month postpartum questionnaire and $60.2 \%$ completing the 36-month postpartum questionnaire. ${ }^{14}{ }^{15}$ Written informed consent was obtained from all participants, and the Regional Committee for Medical Research Ethics and the Norwegian Data Inspectorate approved the study; this analysis was granted an exemption from the University of Massachusetts Medical School Institutional Review Board. Data were taken from the quality-ensured Data V.6, released by MoBa in 2012 and includes all children born before 2009 for whom the age 3 years questionnaire was received by 4 May 2011; these data were linked to the Medical Birth Registry of Norway (MBRN) using participants' 11-digit personal identification numbers. Details of selection into the study are described in figure 1 .

Exclusion criteria were as follows: infant not born alive, multiple births, major congenital malformations or chromosomal abnormalities, and indication of triptan exposure where we were unable to determine whether the triptan was taken prior to or during pregnancy. In total, 7220 women were excluded. We included 59468 mother-child dyads with complete outcome data at the 18-month and/or 36-month follow-up. We conducted a complete case analysis in which dyads with missing data on variables thought to be confounders were excluded, leaving an analytic sample of 50469 women, of which 14790 had complete outcome data only at 18-month follow-up, 6774 had complete data only at 36 months and 28905 had complete outcome data at 18 and 36 months. In analyses of timing of triptan exposure, we included only 5484 women with a self-reported history of migraine headache at the first pregnancy questionnaire.

\section{Triptan exposure}

Information on exposure to medications was gathered prospectively from two prenatal (Q1-gestational week 17, Q3-gestational week 30) and one postpartum (Q4-6 months postpartum) questionnaires. Women indicated when they had taken a medication (during the 6 months before pregnancy, during weeks $0-4,5-8,9-12$ and/or 13 or later for Q1, during weeks 13-16, 17-20, 21-24, 25-28 and/or 29 or later for Q3, and from week 30 until birth for $\mathrm{Q} 4)$ and to write the name of the medication in a text box. Medications were classified according to the WHO Anatomical Therapeutic Chemical (ATC) Classification System. ${ }^{16}$ The ATC code N02CG was used to determine triptan exposure. Triptan medications were further classified into specific compounds: N02CC01 (sumatriptan), N02CC02 (naratriptan), N02CC03 (zolmitriptan), N02CC04 (rizatriptan), N02CC05 (almotriptan), N02CC06 (eletriptan) and N02CC07 (frovatriptan). No information was available on formulation or dose.

Information on migraine was gathered prospectively from pregnancy questionnaire 1 , which asked whether the woman had migraine within 6 months prior to pregnancy or during pregnancy, up to week 17 of pregnancy. Four exposure groups were created: prenatal triptan exposure, prepregnancy triptan use, migraine only and population comparison.

\section{Neurodevelopmental Outcomes Child Behavior Checklist}

The Child Behavior Checklist (CBCL) is a validated measure of child behaviour widely used in clinical and research practice; a shortened version, validated in a Norwegian population, was used in MoBa. ${ }^{17}$ The externalising ('attention problems' and 'aggressive behaviour' subscales) and internalising ('emotionally reactive', 'anxious/depressed' and 'somatic symptoms' subscales) domains were used. Standardised z-scores over or equal to 1.50 on the CBCL measure were used as a clinically relevant cut-off as recommended by Achenbach and Ruffle. $^{18}{ }^{19}$ Children scoring over this cut-off had behavioural problems (externalising or internalising) more extreme than $94 \%$ of the sample. 
Figure 1 Inclusion and exclusion criteria.

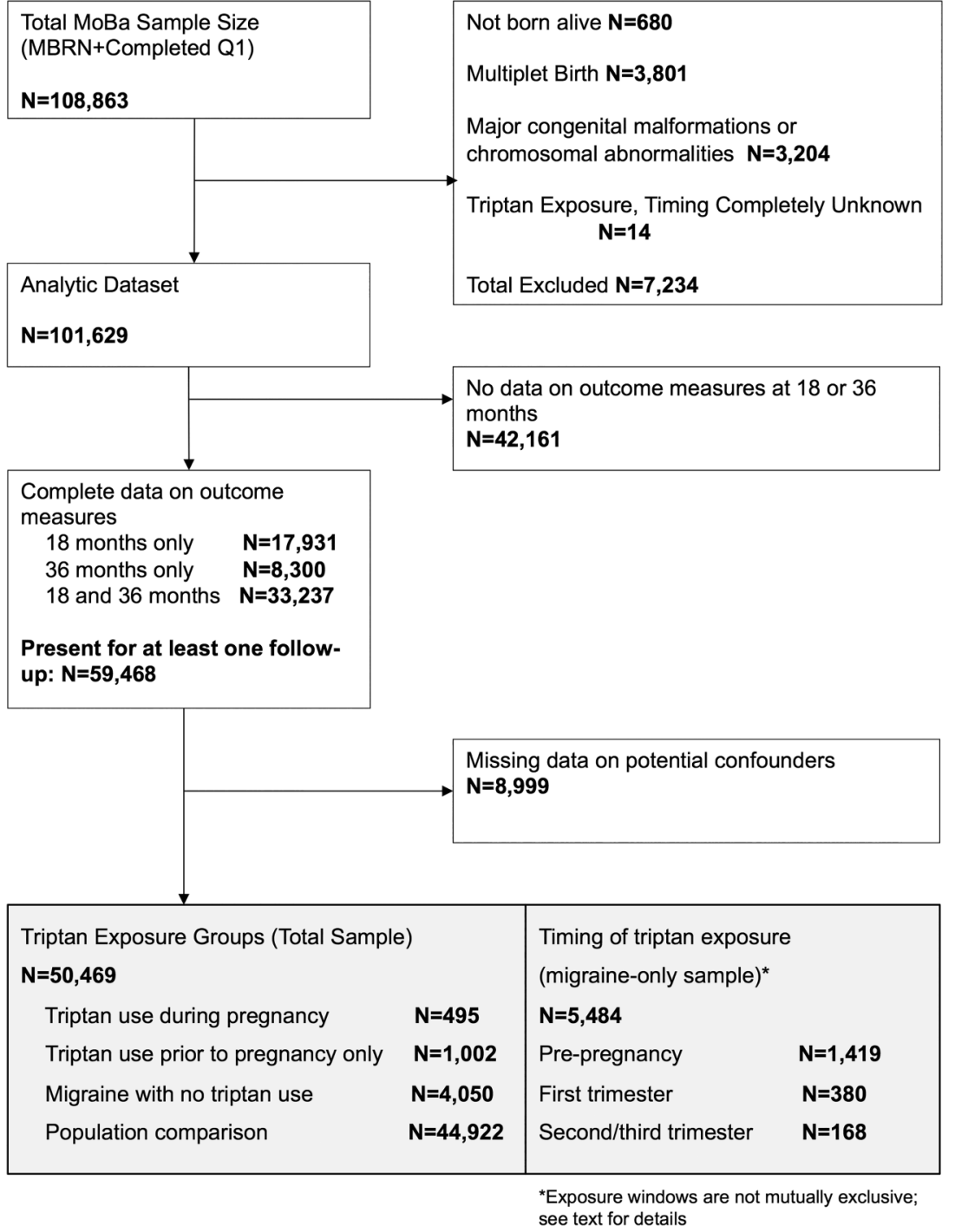

\section{Emotionality, Activity and Shyness Temperament} Questionnaire

The Emotionality, Activity and Shyness Temperament Questionnaire (EAS) measures four temperament domains (emotionality, shyness, sociability and activity). A substantial body of literature has linked earlychildhood temperament to later-life depression and other psychiatric diagnoses. ${ }^{20} 21$ The shortened version of the EAS used in the MoBa study was developed with Norwegian social norms in mind and includes 12 descriptions (eg, "Your child likes to be with people"; "Your child cries easily"), and parents are asked to rate how well each statement applies to their child; in a Norwegian sample, internal consistency $(\alpha)$ within each scale ranged from 0.48 to $0.79 .^{22}$ We calculated z-scores based on the sample distribution of each domain. Higher z-scores indicate greater parental endorsement of each temperament trait (eg, more shy or more sociable) relative to parental reports of other children in the sample. We additionally categorised scores to indicate temperamental traits more extreme than $94 \%$ of the sample (z-score $\geq 1.50$ ).

\section{Ages and Stages Questionnaire}

The Ages and Stages Questionnaire (ASQ) is a parentcompleted questionnaire appropriate for children aged from 4 months to 5 years. Deficits detected by the ASQ are predictive of school difficulties in older children, ${ }^{23}$ and fine motor skills are highly predictive of later academic achievement. ${ }^{24}$ The abbreviated ASQ used in MoBa includes questions about developmental milestone attainment in three major categories: gross motor, fine motor, and communication; this shortened version has been validated in a Norwegian population and had excellent test-retest reliability and agreement between parents and professional examiners. ${ }^{25}$ Scores on the ASQ domains were highly skewed; to address this, we categorised children at each time point as having problems in a given domain if they scored at or above the 94th centile in the sample, versus scoring below the 94th centile. This categorisation is comparable to using a z-score of $\geq 1.50$ as a cut-off.

The CBCL, EAS and ASQ have all been validated in children as young as 18 months. Complete information on the neurodevelopmental outcomes used in this study, 
including procedures used for validation in the MoBa cohort, and the questions selected for each instrument, are available online. ${ }^{26}$

\section{Concomitant medication use}

We examined other pain medications and psychotropic medications as potential confounders, acetaminophen, opioids, non-steroidal anti-inflammatory drugs (NSAIDs), antidepressants, benzodiazepines and anti-epileptics. All comedications were categorised as ever versus never used in pregnancy and prior to pregnancy.

\section{Maternal characteristics}

Maternal age, prepregnancy body mass index (BMI) (underweight or $<18.5 \mathrm{~kg} / \mathrm{m}^{2}$, normal weight or $18.5-$ $25 \mathrm{~kg} / \mathrm{m}^{2}$, or overweight, $>25 \mathrm{~kg} / \mathrm{m}^{2}$ according to WHO guidelines), education (primary or secondary vs university or higher), marital status (married or cohabiting vs other), parity (multiparous vs primiparous) and depression history (yes or no) were all ascertained by selfreport on Q1. Maternal depressive and anxiety symptoms were measured by a validated short version of the Hopkins Symptom Checklist (SCL). ${ }^{27}$ Smoking (ever during pregnancy vs not during pregnancy) and alcohol use (ever during pregnancy vs not during pregnancy) were ascertained by combining information from selfreport as well as linkage to the MBRN.

\section{Loss to follow-up}

We observed substantial loss to follow-up at the 18-month and 36-month questionnaires $(57.3 \%$ present at 18 and 36 months, $29.3 \%$ at 18 months only and $13.4 \%$ at 36 months only). To adjust for the potential for selection bias due to measured predictors of attrition, we constructed stabilised inverse probability of censoring weights (IPCW) and conducted weighted analyses. ${ }^{28}$

\section{Data analysis}

The purpose of this analysis was to examine differences in neurodevelopmental changes over time associated with prenatal triptan exposure. First, we examined descriptive statistics across the four main exposure groups and compared absolute percentages (for categorical measures); we considered a difference of $>5 \%$ to be meaningful. Next, we used generalised estimating equations (GEE) to fit generalised linear models, specifying a binomial distribution and a log link, that included fixed effects for exposure group (prenatal triptan exposure, prepregnancy triptan use, migraine only and population comparison), time (18 and 36 months) and their interaction. GEE models were selected for their approach to missing outcome data (using all available observations, rather than casewise deletion for observations present only at a single time point), as well as their ability to model covariation using flexible covariance structures among repeated measures. Based on comparisons between the empirical and model-based covariance matrices, we selected an exchangeable covariance structure for all models. The resulting estimates represent the change in risk ( $r-R R$ ) of having a clinically meaningful neurodevelopmental outcome between 18 and 36 months of age for children with prenatal triptan exposure, relative to each contrast group (prepregnancy triptan use only, migraine only and population comparison), with 95\% CIs calculated using robust SEs. Models were adjusted for maternal characteristics (age, prepregnancy BMI, parity, marital status, education, smoking or alcohol use in pregnancy, depression symptom severity) and concomitant medication use (NSAIDs, acetaminophen, opioids, antidepressants). To assist in the interpretation of the data, we used model-based predicted probability of outcome at 18 and 36 months to create line graphs of the change in outcome over time for each exposure group. Examination of graphs was useful because the r-RR of $>1$ could be a result of qualitatively different phenomena (eg, greater increased risk over time in triptan group relative to increased risks observed in the contrast group, increased risk in the triptan users group and decreased risk in the comparison group).

Finally, for the neurodevelopmental outcomes that were associated with any triptan use during pregnancy, we developed marginal structural models (MSM) to understand the effect of exposure timing on the difference in change of the outcome from 18 to 36 months. We fit MSM with stabilised inverse probability of treatment weights (IPTW) to adjust for measured confounding by baseline characteristics (maternal age, prepregnancy BMI, sociodemographic variables), time-invariant predictors (smoking and alcohol use during pregnancy, folate supplementation, maternal depression severity) as well as time-varying confounders (other medication use, including acetaminophen, NSAIDs, opioids and antidepressants). The MSM approach results in unbiased effect estimates under assumptions of exchangeability and positivity, and allows for appropriate adjustment for the effects of confounders that are also mediators. ${ }^{29}{ }^{30}$ We created the IPTW via logistic regression at each exposure time point (prepregnancy, first trimester, second/third trimester) created an IPTW equal to the product of the weight from each time point. The product of IPTW and IPCW was used as the total MSM weight, to account for measured confounding and loss to follow-up. Weighted GEE models were then fit within the migraine-only group. Results are given as the change in risk ratio over time (r-RR) for triptan exposure at each time point (prepregnancy, first trimester, second/ third trimester) relative to no exposure during that time, with $95 \%$ CIs estimated using robust SEs to account for clustering induced by weighting. For analyses conducted in the first and second steps, we interpreted a significant time-by-group interaction (95\% CI that did not include 1) to be indicative of a difference in change between exposure groups, from 18 to 36 months of follow-up.

\section{RESULTS}

This study included 50469 women, of whom $1.0 \%$ reported using a triptan at least once during pregnancy, 
$2.0 \%$ used triptans prior to pregnancy only and $8.0 \%$ had a history of migraine with no use of triptans. Among the 5484 women with history of migraine ( $10.9 \%$ of the total sample), $27.1 \%$ used triptans prior to pregnancy, $6.9 \%$ reported use during the first trimester and $3.1 \%$ reported use during the second or third trimester.

Women who reported triptan use during pregnancy differed little from comparison groups in demographic characteristics; age, prepregnancy BMI, parity, educational attainment, smoking during pregnancy and folate supplementation were similar across groups. However, women who used triptans during pregnancy had higher depression and anxiety symptom scores than women who discontinued triptan use ( 0.16 vs 0.03$)$. In addition, women with triptan use during pregnancy were more likely to use other medications at higher rates during pregnancy compared to women who discontinued triptan use, including opioids ( $13.3 \%$ vs $4.6 \%)$, acetaminophen $(78.2 \%$ vs $69.8 \%)$ and NSAIDs $(22.0 \%$ vs $10.1 \%$ ) (table 1).

The Externalising Behaviours subscale of the CBCL, as well as the Emotionality and Activity subscales of the EAS, describes a set of behaviours characterised by increased activity, aggression and emotional reactivity. Children whose mothers used triptans during pregnancy had substantial increased risk of high emotionality compared to static or decreased emotionality in children whose mothers used triptans only prior to pregnancy (r-RR 2.18, 95\% CI 1.03 to 4.53) as well as those with a history of migraine without triptan use (r-RR 2.51, 95\% CI 1.27 to 4.90 ) and a migraine-free population comparison group (r-RR 2.16, 95\% CI 1.14 to 2.14) (table 2, figure 2). When we examined the association of timing of triptan exposure within the group of women with migraine using MSM with inverse probability weights, the r-RR estimate for first trimester exposure relative to no first trimester exposure was 1.54 (95\% CI 0.57 to 4.13) and the r-RR estimate for second/third trimester exposure relative to no second/third trimester exposure was 2.41 (95\% CI 0.71 to 8.20$)$ (table 3 ). We also observed differences in the rate of change for activity: children with prenatal triptan exposure had lesser decreases in activity from 18 to 36 months, relative to the prepregnancy (r-RR $1.70,95 \%$ CI 1.02 to 2.80 ), migraine only (r-RR $1.57,95 \%$ CI 1.04 to 2.36 ) and population comparison (r-RR 1.67, 95\% CI 1.14 to 2.14) groups (table 2). Examining the association between outcome and timing of exposure revealed that the r-RR estimate for second/third trimester exposure relative to no second/third trimester exposure was 1.37 (95\% CI 0.46 to 4.10 ) (table 3). Externalising behaviour in children with prenatal triptan exposure, as measured by the CBCL, remained elevated compared to all contrast groups but did not show evidence of increase or decrease over time (table 2, figure 2).

The Internalising Behaviour subscale of the CBCL, along with the Shyness and Sociability subscales of the
EAS, describes a set of symptoms characterised by anxiety, shyness and withdrawal. Shyness and sociability were not associated with use of triptans during pregnancy (table 2, see online supplementary figure $\mathrm{S} 1$ ).

We saw no differences in change of risk for gross motor, fine motor or communication problems from 18 to 36 months for children with prenatal triptan exposure, relative to any comparison group (table 2, see online supplementary figure S2).

\section{DISCUSSION}

We observed several neurodevelopmental domains in which change in neurodevelopment was substantially different for children with prenatal triptan exposure, including emotionality and activity; these domains appear to be associated with prenatal triptan exposure specifically, rather than migraine. There were no overall differences in internalising behaviours and shyness and motor problems or communication problems associated with either triptan exposure or migraine.

Our previous work, which was the first to report on neurodevelopmental sequelae of prenatal triptan exposure, indicated that exposed children had higher rates of externalising problems at 36 months than those without prenatal exposure. ${ }^{11}$ The findings from the current study suggest that these elevated rates of externalising behaviour remain relatively stable between 18 and 36 months; additionally, the observed increases in emotionality and activity describe a consistent profile of temperamental and behavioural dysregulation associated with prenatal triptan exposure. Several studies in the animal literature suggest that $5-\mathrm{HT}_{1 \mathrm{~B}}$ and $5-\mathrm{HT}_{1 \mathrm{D}}$ receptor expression modifies fetal brain development, ${ }^{10} 31$ providing a plausible biological mechanism for this observed association.

A possible alternative explanation for our findings is that women who used triptans in pregnancy had the most severe migraine, whereas women who discontinued triptan use prior to pregnancy and women with migraine who did not use triptans had less severe illness. Our prior work found that underlying migraine severity explained some, but not all, of the observed associations, ${ }^{12}$ and that confounding by migraine severity would have to be very strong to fully explain our findings. ${ }^{11}$ Therefore, while we cannot rule out confounding by indication, it is unlikely to fully explain these results, which suggest a distinct profile for children with prenatal exposure to triptans in which changes in externalising-type behaviours from 18 to 36 months were markedly different from all comparison groups.

The prevalence of externalising problems among children with prenatal triptan exposure was elevated at 18 months and remained relatively stable at 36 months, while the prevalence of emotionality and activity increased from 18 to 36 months in children with prenatal exposure, compared to all contrast groups. Emotionality, in the context of the Emotionality, Activity 
Table 1 Maternal and pregnancy characteristics

\begin{tabular}{|c|c|c|c|c|}
\hline & \multicolumn{3}{|c|}{ Migraine } & \multirow{2}{*}{$\begin{array}{l}\text { No migraine } \\
\text { Population } \\
\text { comparison } \\
\mathrm{N}=44922 \\
\end{array}$} \\
\hline & $\begin{array}{l}\text { Triptans in } \\
\text { pregnancy } \\
\mathrm{N}=495\end{array}$ & $\begin{array}{l}\text { Triptans prior to } \\
\text { pregnancy } \\
\mathrm{N}=1002\end{array}$ & $\begin{array}{l}\text { No triptan } \\
\text { history } \\
\mathrm{N}=4050\end{array}$ & \\
\hline Age in years (mean, SD) & $30.9(4.3)$ & $30.4(4.1)$ & $30.2(4.5)$ & $30.3(4.4)$ \\
\hline \multicolumn{5}{|l|}{$\mathrm{BMI}\left(\mathrm{kg} / \mathrm{m}^{2}\right)(\mathrm{N}, \%)$} \\
\hline$<18.5$ & $12(2.4)$ & $25(2.5)$ & $145(3.6)$ & $1216(2.7)$ \\
\hline $18.5-25$ & $307(62.0)$ & $608(60.7)$ & $2491(61.5)$ & $29747(66.2)$ \\
\hline$>25$ & $176(35.6)$ & 369 (36.8) & 1414 (34.9) & $13959(31.1)$ \\
\hline Multiparous & $254(51.3)$ & 467 (46.6) & $2204(54.4)$ & $24467(54.5)$ \\
\hline Married or cohabitating & $474(95.8)$ & $980(97.8)$ & 3922 (96.8) & $43761(97.4)$ \\
\hline \multicolumn{5}{|l|}{ Mother education } \\
\hline Primary or secondary & $165(33.3)$ & 339 (33.8) & 1533 (37.9) & $14511(32.3)$ \\
\hline University or higher & $330(66.7)$ & $663(66.2)$ & 2517 (62.2) & $30411(67.7)$ \\
\hline Smoking during pregnancy & $57(11.5)$ & $118(11.8)$ & $552(13.6)$ & $5236(11.7)$ \\
\hline Alcohol during pregnancy & $95(19.2)$ & $146(14.6)$ & $623(15.4)$ & 7506 (16.7) \\
\hline Folate supplementation & $299(60.4)$ & $618(61.7)$ & 2359 (58.3) & $26462(58.9)$ \\
\hline Multivitamin supplementation & $186(37.6)$ & $426(42.5)$ & 1610 (39.8) & $16661(37.1)$ \\
\hline Migraine preventive therapy & $8(1.6)$ & $21(2.1)$ & $22(0.5)$ & $0(0.0)$ \\
\hline \multicolumn{5}{|l|}{ Opioids } \\
\hline Prepregnancy & $40(8.1)$ & $52(5.2)$ & $208(5.1)$ & $609(1.4)$ \\
\hline In pregnancy & $66(13.3)$ & $46(4.6)$ & $188(4.6)$ & $631(1.4)$ \\
\hline \multicolumn{5}{|l|}{ Acetaminophen } \\
\hline Prepregnancy & $233(47.1)$ & $475(47.4)$ & $1794(44.3)$ & $11474(25.5)$ \\
\hline In pregnancy & 387 (78.2) & $699(69.8)$ & $2596(34.1)$ & $19331(43.0)$ \\
\hline \multicolumn{5}{|l|}{ NSAIDs } \\
\hline Prepregnancy & $109(22.0)$ & $247(24.7)$ & $938(23.1)$ & $4450(9.9)$ \\
\hline In pregnancy & $109(22.0)$ & $101(10.1)$ & 509 (12.6) & $2652(5.9)$ \\
\hline \multicolumn{5}{|l|}{ Antidepressants } \\
\hline Prepregnancy & $24(4.9)$ & $48(4.8)$ & 158 (3.9) & $1042(2.3)$ \\
\hline In pregnancy & $11(2.2)$ & $11(1.1)$ & $67(1.7)$ & $426(1.0)$ \\
\hline \multicolumn{5}{|l|}{ Anticonvulsants } \\
\hline Prepregnancy & $2(0.4)$ & $7(0.7)$ & $10(0.3)$ & $41(0.1)$ \\
\hline In pregnancy & $1(0.2)$ & $3(0.3)$ & $13(0.3)$ & $86(0.2)$ \\
\hline \multicolumn{5}{|l|}{ Benzodiazepines } \\
\hline Prepregnancy & $8(1.6)$ & $13(1.3)$ & $38(0.9)$ & $240(0.5)$ \\
\hline In pregnancy & $8(1.6)$ & $5(0.5)$ & $19(0.5)$ & $165(0.4)$ \\
\hline $\begin{array}{l}\text { Maternal depressive/anxiety symptoms* } \\
\text { (mean, SD) }\end{array}$ & $0.16(1.70)$ & $0.03(1.84)$ & $0.23(1.88)$ & $-0.12(1.63)$ \\
\hline Pre-eclampsia & $33(6.7)$ & $47(4.7)$ & $170(4.2)$ & $1536(3.4)$ \\
\hline Small for gestational age & $35(7.1)$ & $65(6.5)$ & $269(6.6)$ & $2737(6.1)$ \\
\hline Apgar $5(<7) \ddagger$ & $7(1.4)$ & $9(0.9)$ & $30(0.7)$ & $415(0.9)$ \\
\hline Preterm§ & $19(3.8)$ & $45(4.5)$ & $193(4.8)$ & $2026(4.5)$ \\
\hline Low birth weight & $11(2.2)$ & $24(2.4)$ & $108(2.7)$ & 1047 (2.3) \\
\hline \multicolumn{5}{|c|}{$\begin{array}{l}\text { Numbers given are frequencies and percents, unless otherwise indicated. } \\
\text { *Average z-score from the SCL; higher positive scores indicate more depressive symptoms. } \\
\text { †Small for gestational age defined as weight below the } 10 \text { th centile for gestational age. } \\
\text { †Number and per cent of children with a } 5 \text {-min Apgar score of }<7 \text {. } \\
\text { §Preterm birth defined as birth before the } 37 \text { th week of gestation. } \\
\text { ILOW birth weight defined as weight at birth below } 2500 \mathrm{~g} \text {, regardless of the gestational age. } \\
\text { BMI, body mass index; NSAIDs, non-steroidal anti-inflammatory drugs; SCL, Symptom Checklist. }\end{array}$} \\
\hline
\end{tabular}

and Shyness Questionnaire, includes items that tap emotional reactivity, while items in the Activity subscale ask about higher levels of physical activity. Of potential interest is the fact that the emotionality and activity domains, as well as the externalising behaviour domain, ask parents to report on observable behaviours in their children, such as temper explosions, hyperactivity and coordination problems, whereas the sociability and shyness domains, as well as internalising behaviours, ask parents to report on their child's internal state. Parents are better reporters of externalising symptoms, whereas children are better reporters of internalising symptoms. ${ }^{32}$ Future studies should include replicating these findings in an older cohort of children, in which child self-report and clinical 
Table 2 Change in neurodevelopmental outcome from 18 to 36 months: change over time for prenatal triptan exposure, relative to prepregnancy triptan use, migraine only and population comparison

\begin{tabular}{|c|c|c|c|c|c|c|}
\hline & \multirow{2}{*}{$\begin{array}{l}\text { Per cent } \\
(18 \text { months }) \dagger\end{array}$} & \multirow{2}{*}{$\begin{array}{l}\text { Per cent } \\
\text { ( } 36 \text { months) }\end{array}$} & \multicolumn{2}{|c|}{ Unadjusted } & \multicolumn{2}{|c|}{$\begin{array}{l}\text { Multivariable } \\
\text { adjusted* }^{*}\end{array}$} \\
\hline & & & r-RR‡ & $95 \% \mathrm{Cl}$ & $\mathbf{r - R R}$ & $95 \% \mathrm{Cl}$ \\
\hline \multicolumn{7}{|l|}{ CBCL externalising behaviour } \\
\hline Prenatal triptan use & 11.0 & 10.0 & & & & \\
\hline Versus prepregnancy triptans only & 7.8 & 6.5 & 1.11 & 0.70 to 1.73 & 1.11 & 0.70 to 1.75 \\
\hline Versus migraine only & 8.1 & 7.6 & 0.99 & 0.69 to 1.43 & 1.00 & 0.70 to 1.43 \\
\hline Versus population comparison & 7.7 & 6.5 & 1.11 & 0.79 to 1.54 & 1.11 & 0.79 to 1.54 \\
\hline \multicolumn{7}{|l|}{ EAS emotionality } \\
\hline Prenatal triptan use & 3.2 & 6.3 & & & & \\
\hline Versus prepregnancy triptans only & 5.3 & 4.7 & 2.18 & 1.03 to 4.57 & 2.18 & 1.03 to 4.53 \\
\hline Versus migraine only & 5.1 & 3.9 & 2.51 & 1.28 to 4.90 & 2.51 & 1.27 to 4.90 \\
\hline Versus population comparison & 5.2 & 4.7 & 2.18 & 1.14 to 4.10 & 2.16 & 1.14 to 4.10 \\
\hline \multicolumn{7}{|l|}{ EAS activity } \\
\hline Prenatal triptan use & 9.2 & 8.3 & & & & \\
\hline Versus prepregnancy triptans only & 9.7 & 4.7 & 1.68 & 1.02 to 2.80 & 1.70 & 1.02 to 2.80 \\
\hline Versus migraine only & 9.8 & 5.3 & 1.57 & 1.05 to 2.34 & 1.57 & 1.04 to 2.36 \\
\hline Versus population comparison & 9.8 & 5.1 & 1.67 & 1.14 to 2.41 & 1.67 & 1.14 to 2.42 \\
\hline \multicolumn{7}{|l|}{ CBCL internalizing behaviour } \\
\hline Prenatal triptan use & 8.1 & 9.5 & & & & \\
\hline Versus prepregnancy triptans only & 6.2 & 10.8 & 0.70 & 0.41 to 1.16 & 0.69 & 0.41 to 1.14 \\
\hline Versus migraine only & 8.7 & 10.5 & 1.01 & 0.66 to 1.57 & 1.02 & 0.66 to 1.57 \\
\hline Versus population comparison & 7.5 & 8.1 & 1.14 & 0.75 to 1.72 & 1.12 & 0.74 to 1.70 \\
\hline \multicolumn{7}{|l|}{ EAS shyness } \\
\hline Prenatal triptan use & 4.9 & 12.3 & & & & \\
\hline Versus prepregnancy triptans only & 3.5 & 9.6 & 0.93 & 0.53 to 1.65 & 0.92 & 0.52 to 1.63 \\
\hline Versus migraine only & 4.0 & 7.7 & 1.30 & 0.81 to 2.08 & 1.30 & 0.81 to 2.08 \\
\hline Versus population comparison & 4.5 & 8.1 & 1.40 & 0.91 to 2.16 & 1.40 & 0.91 to 2.16 \\
\hline \multicolumn{7}{|l|}{ EAS sociability } \\
\hline Prenatal triptan use & 8.8 & 6.3 & & & & \\
\hline Versus prepregnancy triptans only & 6.8 & 6.4 & 0.77 & 0.45 to 1.38 & 0.78 & 0.44 to 1.38 \\
\hline Versus migraine only & 9.3 & 6.1 & 1.12 & 0.69 to 1.80 & 1.13 & 0.70 to 1.82 \\
\hline Versus population comparison & 8.0 & 5.7 & 1.02 & 0.65 to 1.62 & 1.03 & 0.65 to 1.63 \\
\hline \multicolumn{7}{|l|}{ ASQ gross motor } \\
\hline Prenatal triptan use & 1.6 & 1.2 & & & & \\
\hline Versus prepregnancy triptans only & 3.3 & 3.0 & 0.86 & 0.23 to 3.20 & 0.86 & 0.23 to 3.19 \\
\hline Versus migraine only & 2.3 & 3.0 & 0.58 & 0.16 to 2.02 & 0.58 & 0.17 to 2.03 \\
\hline Versus population comparison & 2.3 & 3.1 & 0.55 & 0.16 to 1.87 & 0.55 & 0.16 to 1.88 \\
\hline \multicolumn{7}{|l|}{ ASQ fine motor } \\
\hline Prenatal triptan use & 13.9 & 9.5 & & & & \\
\hline Versus prepregnancy triptans only & 11.6 & 9.4 & 0.84 & 0.52 to 1.36 & 0.85 & 0.52 to 1.37 \\
\hline Versus migraine only & 11.5 & 9.2 & 0.85 & 0.56 to 1.28 & 0.85 & 0.56 to 1.29 \\
\hline Versus population comparison & 11.8 & 10.4 & 0.78 & 0.52 to 1.13 & 0.77 & 0.52 to 1.14 \\
\hline \multicolumn{7}{|l|}{ ASQ communication } \\
\hline Prenatal triptan use & 3.4 & 4.6 & & & & \\
\hline Versus prepregnancy triptans only & 4.1 & 4.5 & 1.20 & 0.55 to 2.64 & 1.22 & 0.56 to 2.68 \\
\hline Versus migraine only & 3.8 & 5.2 & 0.96 & 0.48 to 1.93 & 0.97 & 0.48 to 1.95 \\
\hline Versus population comparison & 4.1 & 5.0 & 1.09 & 0.56 to 2.16 & 1.12 & 0.57 to 2.19 \\
\hline \multicolumn{7}{|c|}{$\begin{array}{l}\text { “Models are adjusted for maternal age, prepregnancy BMI, parity, marital status, education, smoking or alcohol use during pregnancy, SCL } \\
\text { depression/anxiety severity score and concomitant medication use during pregnancy (acetaminophen, opioids, NSAIDs, antidepressants). } \\
\text { tPer cent is the per cent with outcome, respectively, at each measurement (18 and } 36 \text { months postpartum): for example, at } 18 \text { months, } 11 \% \\
\text { of children with prenatal triptan exposure had externalising symptoms at or above a z-score of } 1.50 \text {, compared with } 7.8 \% \text { of children whose } \\
\text { mothers used triptans prior to pregnancy. } \\
\text { fr-RR is the group-by-time interaction coefficient from the generalised estimating equation model; it is the difference in change from } 18 \text { to } \\
36 \text { months for prenatal triptan exposure, relative to each contrast group. } \\
\text { ASQ, Ages and Stages Questionnaire; BMI, body mass index; CBCL, Child Behaviour Checklist; EAS, Emotionality, Activity and Shyness } \\
\text { Temperament Questionnaire; NSAIDs, non-steroidal anti-inflammatory drugs; r-RR, ratio of risk ratios; SCL, Symptom Checklist. }\end{array}$} \\
\hline
\end{tabular}




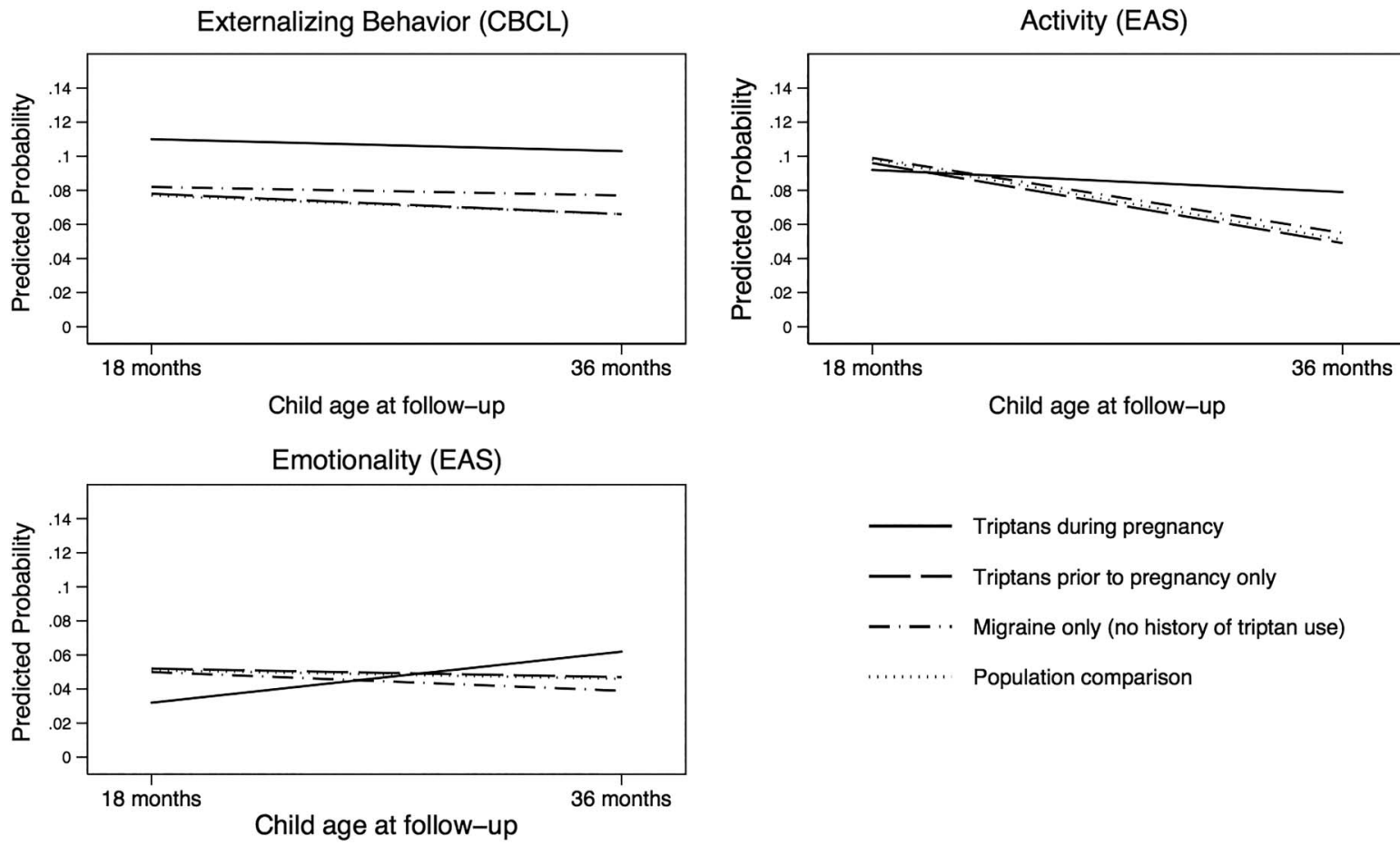

Figure 2 Changes from 18 to 36 months for externalising-type behaviours.

Table 3 Change in neurodevelopmental outcome from 18 to 36 months: change over time associated with timing of triptan exposure, within migraine-only sample $(\mathrm{N}=5484)$

\begin{tabular}{|c|c|c|c|c|c|c|}
\hline & \multirow[b]{2}{*}{ Per cent (8 months) $\dagger$} & \multirow[b]{2}{*}{ Per cent (36 months) } & \multicolumn{2}{|c|}{ Unadjusted } & \multicolumn{2}{|c|}{ MSM $^{*}$} \\
\hline & & & $\overline{\text { r-RR‡ }}$ & $95 \% \mathrm{Cl}$ & $\overline{r-R R}$ & $95 \% \mathrm{Cl}$ \\
\hline \multicolumn{7}{|c|}{ CBCL externalising behaviour } \\
\hline Prepregnancy & 8.4 & 7.5 & 0.94 & 0.67 to 1.30 & 0.90 & 0.64 to 1.25 \\
\hline First trimester & 11.6 & 11.1 & 1.16 & 0.73 to 1.86 & 1.01 & 0.56 to 1.80 \\
\hline Second/third trimester & 10.7 & 9.8 & 0.96 & 0.48 to 1.91 & 0.50 & 0.17 to 1.48 \\
\hline \multicolumn{7}{|l|}{ EAS emotionality } \\
\hline Prepregnancy & 4.7 & 4.7 & 1.07 & 0.69 to 1.67 & 1.07 & 0.68 to 1.67 \\
\hline First trimester & 3.8 & 5.6 & 1.43 & 0.58 to 3.51 & 1.54 & 0.57 to 4.13 \\
\hline Second/third trimester & 4.0 & 7.1 & 1.52 & 0.48 to 4.85 & 2.41 & 0.71 to 8.20 \\
\hline \multicolumn{7}{|l|}{ EAS activity } \\
\hline Prepregnancy & 9.6 & 5.7 & 0.90 & 0.63 to 1.28 & 0.88 & 0.61 to 1.28 \\
\hline First trimester & 9.3 & 8.9 & 1.56 & 0.90 to 2.71 & 1.98 & 0.90 to 4.34 \\
\hline Second/third trimester & 9.4 & 10.7 & 1.56 & 0.76 to 3.21 & 1.37 & 0.46 to 4.10 \\
\hline
\end{tabular}

${ }^{*}$ Per cent is the per cent with outcome at each measurement (18 and 36 months postpartum), among those with exposure at each time point (eg, per cent with externalising problems at 18 months with prenatal triptan use $=\{(75 / 1002) \times 100\}=7.49 \%$. Windows of triptan exposure are not mutually exclusive).

$\mathrm{tr}$-RR is the group-by-time interaction coefficient from the generalised estimating equation model; it is the difference in change from 18 to 36 months for each group, relative to no exposure during that time point.

$¥$ Models are weighted by the product of stabilised IPCW and IPTW; IPCW is the probability of dropout, conditional on maternal age, prepregnancy $\mathrm{BMI}$, marital status, parity, migraine history and use of triptans prior to and during pregnancy. IPTW includes baseline covariates (maternal age, prepregnancy BMI, sociodemographic variables), time-invariant predictors (smoking and alcohol use during pregnancy, folate supplementation, maternal depression severity), time-varying concomitant medication use (acetaminophen, NSAIDs, opioids, antidepressants) and treatment history (triptan use).

BMI, body mass index; CBCL, Child Behaviour Checklist; EAS, Emotionality, Activity and Shyness Temperament Questionnaire; IPCW, inverse probability of censoring weight; IPTW, inverse probability of treatment weight; MSM, marginal structural models; r-RR, ratio of risk ratios.

observation of psychological or behavioural problems are available.

The results of this study have important implications for clinicians who may see children born to mothers with migraine headache. Numerous studies have shown that early-childhood emotional and behavioural problems are predictive of academic and emotional difficulties in adolescence, ${ }^{33-36}$ but that early intervention can effectively 
reduce these problems. ${ }^{37} 38$ Children whose mothers have a history of migraine, and particularly those whose mothers took triptans during pregnancy, may benefit from additional monitoring and potentially, treatment.

There are several important limitations to consider when evaluating the findings from this study. First, MoBa does not collect information on migraine severity, and women with more severe migraine are more likely to use triptans. In our previous research, we carried out sensitivity analyses to examine the potential impact of unmeasured confounding by migraine severity ${ }^{11}$ as well as using an external validation study to calibrate effect estimates $^{12}$ to address this source of confounding, and found that migraine severity would have to be an extremely strong confounder to fully explain our results. However, confounding by indication is difficult to control $^{39}$ and cannot be ruled out as an explanation for the observed results. Second, no information was available on triptan formulation or dose; additionally, we had insufficient power to analyse specific triptans. Triptans have different pharmacokinetic properties and affinities for subclasses of serotonin receptors, ${ }^{40}$ and considering these medications as a class may elide important information on compound-specific risks. Exposure misclassification is possible; however, it is unlikely to be differential with respect to outcome, and so is more likely to have produced bias towards the null, as has been shown in previous validation studies in the MoBa cohort. ${ }^{41}$

These limitations are balanced by important strengths: first, we used advanced analytic methods to appropriately adjust for time-varying confounding by concomitant medication use. Triptan exposure changes over time, and women who use triptans in pregnancy also use many other medications, several of which have previously been associated with neurodevelopmental problems in children. $^{42-44}$ Failure to appropriately adjust for these medications may result in incorrectly attributing effects to triptan exposure that are in fact due to other medications. Our study was set in a large, prospective birth cohort with data available on over-the-counter and prescription medication use, allowing for careful consideration of concomitant medication use, as well as other important confounders such as severity of maternal depressive and anxiety symptoms.

This study suggests that prenatal exposure to migraine and triptans may be associated with neurodevelopmental problems in children. However, these findings and our related work are based on a single cohort and should be replicated in other cohorts before clinical recommendations for treatment of migraine in pregnancy are changed.

\footnotetext{
Author affiliations

${ }^{1}$ The University of Oslo School of Pharmacy, Oslo, Norway

${ }^{2}$ The University of Massachusetts Medical School, Worcester, Massachusetts, USA

${ }^{3}$ PharmacoEpidemiology and Drug Safety Research Group, Department of Pharmacy, University of Oslo, Oslo, Norway

${ }^{4}$ The University of Oslo School of Pharmacy and the Norwegian Institute of Public Health, Oslo, Norway
}

Twitter Follow Hedvig Nordeng at @Pharma_Nordeng

Contributors MEW designed the study and statistical analysis plan, performed statistical analyses, and drafted the manuscript. She is the guarantor. KLL and HMEN consulted on study design and statistical methods, and contributed to the manuscript. JAF contributed to study design development and revised the draft paper.

Funding The Norwegian Mother and Child Cohort Study (MoBa) is supported by the Norwegian Ministry of Health and the Ministry of Education and Research, NIH/NIEHS (contract number N01-ES-75558), NIH/NINDS (grant numbers 1 U01 NS 047537-01 and 2 U01 NS 047537-06A1).

Competing interests KLL has consulted with Janssen Pharmaceuticals on antipsychotic medications and schizophrenia in the dually eligible population as well as Glaxo Smith Kline on methodological work related to patient-centred outcomes in lupus. JAF has received research support from Alcobra, Glaxo Smith Kline, Pfizer, Neuren, Roche, Seaside Therapeutics, SyneuRX International, as well as NIMH, NICHD and NINDS. In addition, she serves on a data safety monitoring board for a Forest Pharmaceuticals clinical trial.

Ethics approval Regional Committee for Medical Research Ethics and the Norwegian Data Inspectorate provided ethics approval for this study.

Provenance and peer review Not commissioned; externally peer reviewed.

Data sharing statement No additional data are available.

Open Access This is an Open Access article distributed in accordance with the Creative Commons Attribution Non Commercial (CC BY-NC 4.0) license, which permits others to distribute, remix, adapt, build upon this work noncommercially, and license their derivative works on different terms, provided the original work is properly cited and the use is non-commercial. See: http:// creativecommons.org/licenses/by-nc/4.0/

\section{REFERENCES}

1. MacGregor EA. Headache in pregnancy. Neurol Clin 2012;30: 835-66.

2. Lipton RB, Bigal ME, Diamond M, et al. Migraine prevalence, disease burden, and the need for preventive therapy. Neurology 2007;68:343-9.

3. Chu MK, Buse DC, Bigal ME, et al. Factors associated with triptan use in episodic migraine: results from the American Migraine Prevalence and Prevention Study. Headache 2012;52:213-23.

4. Amundsen $\mathrm{S}$, Nordeng $\mathrm{H}$, Nezvalová-Henriksen $\mathrm{K}$, et al. Pharmacological treatment of migraine during pregnancy and breastfeeding — current evidence and practical recommendations. Nat Rev Neurol 2015;11:209-19.

5. Nezvalová-Henriksen K, Spigset O, Nordeng H. Triptan exposure during pregnancy and the risk of major congenital malformations and adverse pregnancy outcomes: results from the Norwegian Mother and Child Cohort Study. Headache 2010;50:563-75.

6. Källén B, Nilsson E, Olausson PO, et al. Delivery outcome after maternal use of drugs for migraine. Drug Saf 2011;34:691-703.

7. Duong S, Nordeng H, Einarson A, et al. Safety of triptans for migraine headaches during pregnancy and breastfeeding. Can Fam Physician 2010;56:537-9.

8. Marchenko A, Etwel F, Olutunfese O, et al. Pregnancy outcome following prenatal exposure to triptan medications: a meta-analysis. Headache 2015;55:490-501.

9. Bonnin A, Levitt P. Fetal, maternal, and placental sources of serotonin and new implications for developmental programming of the brain. Neuroscience 2011;197:1-7.

10. Bonnin A, Torii M, Wang L, et al. Serotonin modulates the response of embryonic thalamocortical axons to netrin-1. Nat Neurosci 2007;10:588-97.

11. Wood ME, Lapane K, Frazier JA, et al. Prenatal triptan exposure and internalising and externalising behaviour problems in 3-year-old children: results from the Norwegian Mother and Child Cohort Study. Paediatr Perinat Epidemiol 2016;30:190-200.

12. Wood ME, Frazier JA, Nordeng HME, et al. Prenatal triptan exposure and parent-reported early childhood neurodevelopmental outcomes: an application of propensity score calibration to adjust for unmeasured confounding by migraine severity. Pharmacoepidemiol Drug Saf 2015;25:493-502.

13. Magnus $\mathrm{P}$, Birke $\mathrm{C}$, Vejrup K, et al. Cohort Profile Update: The Norwegian Mother and Child Cohort Study (MoBa). Int J Epidemiol 2016;45:382-8 
14. Magnus P. The Norwegian Mother and Child Cohort Study (MoBa)new research possibilities. Nor Epidemiol 2007;17:107-10.

15. Stoltenberg C, Schjølberg S, Bresnahan M, et al. The Autism Birth Cohort: a paradigm for gene-environment-timing research. $\mathrm{Mol}$ Psychiatry 2010;15:676-80.

16. World Health Organization. The Anatomical Therapeutic Chemical Classification System with Defined Daily Doses (ATC/ DDD.) Geneva: WHO,2012. http://www.who.int/classifications/atcddd/en

17. Nøvik TS. Validity of the child behaviour checklist in a Norwegian sample. Eur Child Adolesc Psychiatry 1999;8:247-54.

18. Achenbach TM. Manual for the child behavior checklist/2-3. Burlington (VT): Univ. Vermont, Dep. Psychiatry, 1992.

19. Achenbach TM, Ruffle TM. The Child Behavior Checklist and related forms for assessing behavioral/emotional problems and competencies. Pediatr Rev 2000;21:265-71.

20. Klein DN, Kotov R, Bufferd SJ. Personality and depression: explanatory models and review of the evidence. Annu Rev Clin Psychol 2011;7:269-95.

21. Althoff RR, Rettew DC, Faraone SV, et al. Latent class analysis shows strong heritability of the child behavior checklist-juvenile bipolar phenotype. Biol Psychiatry 2006;60:903-11.

22. Mathiesen KS, Tambs K. The EAS Temperament Questionnairefactor structure, age trends, reliability, and stability in a Norwegian sample. J Child Psychol Psychiatry 1999;40:431-9.

23. Halbwachs M, Muller JB, Nguyen The Tich S, et al. Predictive value of the parent-completed ASQ for school difficulties in preterm-born children $<35$ weeks' GA at five years of age. Neonatology 2014;106:311-6.

24. Grissmer D, Grimm KJ, Aiyer SM, et al. Fine motor skills and early comprehension of the world: two new school readiness indicators. Dev Psychol 2010;46:1008-17.

25. Richter J, Janson H. A validation study of the Norwegian version of the Ages and Stages Questionnaires. Acta Paediatr 2007;96:748-52.

26. Questionnaires from $\mathrm{MoBa}$. Nor Inst Public Heal, 2016. https://www. fhi.no/en/migrering/english/mainmenu/studies/ mother-and-child-cohort-study/questionnaire/ questionnaires-from-moba/ (accessed 24 Jun 2016).

27. Strand $\mathrm{BH}$, Dalgard OS, Tambs K, et al. Measuring the mental health status of the Norwegian population: a comparison of the instruments SCL-25, SCL-10, SCL-5 and MHI-5 (SF-36). Nord J Psychiatry 2003;57:113-18.

28. Cole SR, Hernán MA. Constructing inverse probability weights for marginal structural models. Am J Epidemiol 2008;168:656-64.

29. Hernán MA, Brumback B, Robins JM. Marginal structural models to estimate the causal effect of zidovudine on the survival of HIV-positive men. Epidemiology 2000;11:561-70.

30. Robins JM. Association, causation, and marginal structural models. Synthese 1999;121:151-79.
31. Bonnin A, Levitt P. Placental source for $5-\mathrm{HT}$ that tunes fetal brain development. Neuropsychopharmacology 2012;37:299-300.

32. Sourander A, Helstelä L, Helenius H. Parent-adolescent agreement on emotional and behavioral problems. Soc Psychiatry Psychiatr Epidemiol 1999;34:657-63.

33. Beyer T, Postert C, Müller JM, et al. Prognosis and continuity of child mental health problems from preschool to primary school: results of a four-year longitudinal study. Child Psychiatry Hum Dev 2012;43:533-43.

34. Holtmann M, Buchmann AF, Esser G, et al. The Child Behavior Checklist-Dysregulation Profile predicts substance use, suicidality, and functional impairment: a longitudinal analysis. J Child Psychol Psychiatry 2011;52:139-47.

35. Pihlakoski L, Sourander A, Aromaa M, et al. The continuity of psychopathology from early childhood to preadolescence: a prospective cohort study of 3-12-year-old children. Eur Child Adolesc Psychiatry 2006;15:409-17.

36. Mathyssek CM, Olino TM, Verhulst FC, et al. Childhood internalizing and externalizing problems predict the onset of clinical panic attacks over adolescence: the TRAILS study. PLoS One 2012;7:e51564.

37. Feinfield KA, Baker BL. Empirical support for a treatment program for families of young children with externalizing problems. J Clin Child Adolesc Psychol 2004;33:182-95.

38. Bagner DM, Sheinkopf SJ, Vohr BR, et al. Parenting intervention for externalizing behavior problems in children born premature: an initial examination. J Dev Behav Pediatr 2010;31:209-16.

39. Bosco JLF, Silliman RA, Thwin SS, et al. A most stubborn bias: no adjustment method fully resolves confounding by indication in observational studies. J Clin Epidemiol 2010;63: $64-74$

40. Jhee SS, Shiovitz T, Crawford AW, et al. Pharmacokinetics and pharmacodynamics of the triptan antimigraine agents: a comparative review. Clin Pharmacokinet 2001;40:189-205.

41. Skurtveit S, Selmer R, Tverdal A, et al. Drug exposure: inclusion of dispensed drugs before pregnancy may lead to underestimation of risk associations. J Clin Epidemiol 2013;66:964-72.

42. Brandlistuen RE, Ystrom E, Nulman I, et al. Prenatal paracetamol exposure and child neurodevelopment: a sibling-controlled cohort study. Int J Epidemiol 2013;42:1702-13.

43. Liew Z, Ritz B, Rebordosa C, et al. Acetaminophen use during pregnancy, behavioral problems, and hyperkinetic disorders. JAMA Pediatr 2014;168:313-20.

44. Pedersen LH, Henriksen TB, Bech BH, et al. Prenatal antidepressant exposure and behavioral problems in early childhood-a cohort study. Acta Psychiatr Scand 2013;127: 126-35. 